\title{
On the analysis of the Illumina 450K array data: probes ambiguously mapped to the human genome
}

\author{
Xu Zhang ${ }^{1}$, Wenbo $\mathrm{Mu}^{2}$ and Wei Zhang ${ }^{3,4,5 *}$ \\ Department of Ecology and Evolution, University of Chicago, Chicago, IL, USA \\ 2 Department of Bioengineering, University of Illinois at Chicago, Chicago, IL, USA \\ ${ }^{3}$ Department of Pediatrics, University of Illinois at Chicago, Chicago, IL, USA \\ ${ }^{4}$ Institute of Human Genetics, University of Illinois at Chicago, Chicago, IL, USA \\ 5 University of Illinois Cancer Center, Chicago, IL, USA \\ *Correspondence:weizhan1@uic.edu
}

\section{A commentary on}

The impact of recent alcohol use on genome wide DNA methylation signatures

by Philibert, R. A., Plume, J. M., Gibbons, F.X., Brody, G. H., and Beach, S. R. (2012). Front. Genet. 3:54. doi: 10.3389/fgene.2012.00054

The newly developed Illumina HumanMethylation450 BeadChip (450K array; Illumina, Inc., San Diego, CA, USA) allows unprecedented genome-wide profiling of DNA methylation at $>450,000 \mathrm{CpG}$ and non-CpG methylation sites (Sandoval et al., 2011). Utilizing the $450 \mathrm{~K}$ array, Philibert et al. (2012) examined the relationship of recent alcohol intake to genome-wide methylation patterns in lymphoblast DNA samples derived from 165 female subjects participating in the Iowa Adoption Studies. The authors' interesting paper demonstrated that the $450 \mathrm{~K}$ array could be a useful tool for ongoing and newly designed epigenome projects. However, given the unique design of the platform (for detailed annotations for the $450 \mathrm{~K}$ array including probe sequences: http://www.illumina.com/), some cautions might need to be exerted when analyzing the $450 \mathrm{~K}$ array data, in addition to some general challenges for analyzing the whole-genome DNA methylation data (Laird, 2010). Particularly, we found that a substantial proportion of the $>450,000$ DNA methylation probes on the $450 \mathrm{~K}$ array are not aligned to unique, unambiguous loci in the human genome (Moen et al., 2012). In total, we found $\sim 140,000$ methylation probes ambiguously mapped to multiple locations in the human genome (hg19) with up to two mismatches in the probe sequences using Bowtie (v2.0.0 beta2; Langmead et al., 2009; Langmead and Salzberg, 2012). Briefly, Bowtie is an ultrafast, memory-efficient short read aligner by indexing the genome with an extended Burrows-Wheeler technique, which implements a novel quality-aware backtracking algorithm that permits mismatches (Langmead et al., 2009; Langmead and Salzberg, 2012). Different alignment algorithms, e.g., BLAT (Kent, 2002) and MAQ (Li et al., 2008), would provide similar estimates (unpublished data). In comparison, $\sim 1,000$ methylation probes were found to be ambiguously mapped to the human genome hg18 in the earlier 27K Illumina Human Methylation array $(27 \mathrm{~K}$ array; Bell et al., 2011). Because the much more comprehensive $450 \mathrm{~K}$ array covers not only promoters, but also gene bodies, untranslated regions (UTRs) and "open sea" methylation sites, the problem of ambiguous alignment may particularly need to be taken into account when analyzing the data from this new platform. Notably, $20 \mathrm{CpG}$ methylation probes (e.g., cg24023553 in Table 2; cg00004209 in Table 3; cg24675557 in Table 5) out of the 90 top-ranking probes reported by Philibert et al. (2012) were mapped to ambiguous loci in the current human reference (hg19) using Bowtie (Langmead et al., 2009; Langmead and Salzberg, 2012). Since the problem of ambiguous alignment to the human genome may cause unreliable measurement of DNA methylation level at a particular methylation site, considering this unique problem for this platform may not only facilitate the data analysis (e.g., by improving the multipletesting problem by removing those affected probes), but also help interpret the results by focusing on more reliable biological signals. In addition, other factors (e.g., polymorphisms in the target sequences, potential batch effects) that may affect other platforms (e.g., the 27K array; Bell et al., 2011; Fraser et al., 2012) as well may also need to be considered in the analysis of these data.

\section{ACKNOWLEDGMENT}

This work was supported, in part, by a grant, R21HG006367 (to Wei Zhang) from the NHGRI/NIH.

\section{REFERENCES}

Bell, J. T., Pai, A. A., Pickrell, J. K., Gaffney, D. J., PiqueRegi, R., Degner, J. F., Gilad, Y., and Pritchard, J. K. (2011). DNA methylation patterns associate with genetic and gene expression variation in HapMap cell lines. Genome Biol. 12, R10.

Fraser, H. B., Lam, L. L., Neumann, S. M., and Kobor, M. S. (2012). Population-specificity of human DNA methylation. Genome Biol. 13, R8.

Kent, W. J. (2002). BLAT - the BLAST-like alignment tool. Genome Res. 12, 656-664.

Laird, P. W. (2010). Principles and challenges of genomewide DNA methylation analysis. Nat. Rev. Genet. 11, 191-203.

Langmead, B., and Salzberg, S. L. (2012). Fast gapped-read alignment with Bowtie 2. Nat. Methods 9, 357-359.

Langmead, B., Trapnell, C., Pop, M., and Salzberg, S. L. (2009). Ultrafast and memory-efficient alignment of short DNA sequences to the human genome. Genome Biol. 10, R25.

Li, H., Ruan, J., and Durbin, R. (2008). Mapping short DNA sequencing reads and calling variants using mapping quality scores. Genome Res. 18, 1851-1858.

Moen, L. E., Mu, W., Delaney, S., Wing, C., McQuade, J., Godley, L. A., Dolan, M. E., and Zhang, W. (2012). Differences in DNA methylation between the African and European HapMap populations. Proc. Am. Assoc. Cancer Res. 5010. [Abstract]

Philibert, R.A., Plume, J. M., Gibbons, F. X., Brody, G. H., and Beach, S. R. (2012). The impact of recent alcohol use on genome wide DNA methylation signatures. Front. Genet. 3:54. doi: 10.3389/fgene.2012.00054

Sandoval, J., Heyn, H., Moran, S., Serra-Musach, J., Pujana, M. A., Bibikova, M., and Esteller, M. (2011). Validation of a DNA methylation microarray for 450,000 CpG sites in the human genome. Epigenetics $6,692-702$.

Received: 23 March 2012; accepted: 15 April 2012; published online: 04 May 2012.

Citation: Zhang $X, M u W$ and Zhang $W$ (2012) On the analysis of the Illumina $450 \mathrm{~K}$ array data: probes ambiguously mapped to the human genome. Front. Gene. 3:73. doi: 10.3389/fgene. 2012.00073

This article was submitted to Frontiers in Behavioral and Psychiatric Genetics, a specialty of Frontiers in Genetics.

Copyright $\odot 2012$ Zhang, Mu and Zhang. This is an openaccess article distributed under the terms of the Creative Commons Attribution Non Commercial License, which permits non-commercial use, distribution, and reproduction in other forums, provided the original authors and source are credited. 